\title{
Quench dynamics and defect production in the Kitaev and extended Kitaev models
}

\author{
Shreyoshi Mondal ${ }^{1}$, Diptiman Sen ${ }^{2}$ and K. Sengupta ${ }^{1}$ \\ 1 TCMP division, Saha Institute of Nuclear Physics, 1/AF Bidhannagar, Kolkata 700 064, India \\ 2 Center for High Energy Physics, Indian Institute of Science, Bangalore, 560 012, India
}

(Dated: October 27, 2018)

\begin{abstract}
We study quench dynamics and defect production in the Kitaev and the extended Kitaev models. For the Kitaev model in one dimension, we show that in the limit of slow quench rate, the defect density $n \sim 1 / \sqrt{\tau}$ where $1 / \tau$ is the quench rate. We also compute the defect correlation function by providing an exact calculation of all independent non-zero spin correlation functions of the model. In two dimensions, where the quench dynamics takes the system across a critical line, we elaborate on the results of earlier work [K. Sengupta, D. Sen and S. Mondal, Phys. Rev. Lett. 100, 077204 (2008).] to discuss the unconventional scaling of the defect density with the quench rate. In this context, we outline a general proof that for a $d$ dimensional quantum model, where the quench takes the system through a $d-m$ dimensional gapless (critical) surface characterized by correlation length exponent $\nu$ and dynamical critical exponent $z$, the defect density $n \sim 1 / \tau^{m \nu /(z \nu+1)}$. We also discuss the variation of the shape and the spatial extent of the defect correlation function with the change of both the rate of quench and the model parameters and compute the entropy generated during such a quench process. Finally, we study the defect scaling law, entropy generation and defect correlation function of the two-dimensional extended Kitaev model.
\end{abstract}

PACS numbers: 73.43.Nq, 05.70.Jk, 64.60.Ht, 75.10.Jm

\section{INTRODUCTION}

Quantum phase transitions involve a fundamental change in the symmetry of the ground state of a quantum system. Such a transition usually takes place due to the variation of some parameter $\lambda$ in the Hamiltonian of the system and is necessarily accompanied by diverging length and time scales ${ }^{1}$. A direct consequence of such a diverging time scale is that a quantum system fails to be in the adiabatic limit when it is sufficiently close to the quantum critical point. Thus a time evolution of the parameter $\lambda$ at a finite rate $1 / \tau$, which takes such a system across a quantum critical point located at $\lambda=\lambda_{c}$, leads to failure of the system to follow the instantaneous ground state in a finite region around $\lambda_{c}$. As a result, the state of the system after such a time evolution does not conform to the ground state of its final Hamiltonian leading to the production of defects 2,3 . It is well known that for a slow quench, the density of these defects $n$ depends on the quench time $\tau$ according to $n \sim 1 / \tau^{d \nu /(\nu z+1)}$, where $\nu$ and $z$ are the correlation length and the dynamical critical exponents characterizing the critical point $\underline{4,5,6}$. A theoretical study of such a quench dynamics requires a knowledge of the excited states of the system. As a result, early studies of the quench problem are mostly restricted to quantum phase transitions in exactly solvable models such as the onedimensional (1D) Ising model in a transverse field ${ }^{7,8.9}$, the infinite range ferromagnetic Ising model ${ }^{10}$, the $1 \mathrm{D}$ $\mathrm{XY}$ model ${ }^{11,12}$, and quantum spin chains ${ }^{13,14,15}$. On the experimental side, trapped ultracold atoms in optical lattices provide possibilities of realization of many of the above-mentioned systems ${ }^{16}$. Experimental studies of defect production due to quenching of the magnetic field in a spin-one Bose condensate has also been undertaken ${ }^{17}$.
Recently, Kitaev proposed a 2D spin-1/2 model on a honeycomb lattice with a Hamiltonian 18

$$
\begin{gathered}
H_{1}=\sum_{j+l=\text { even }}\left(J_{1} \sigma_{j, l}^{x} \sigma_{j+1, l}^{x}+J_{2} \sigma_{j-1, l}^{y} \sigma_{j, l}^{y}\right. \\
\left.+J_{3} \sigma_{j, l}^{z} \sigma_{j, l+1}^{z}\right),
\end{gathered}
$$

where $j$ and $l$ denote the column and row indices of the honeycomb lattice. This model has several interesting features which led to a plethora of theoretical works on it $19,22,23$. For example, it provides a rare example where a $2 \mathrm{D}$ model can be exactly solved using a JordanWigner transformation 18,19,20,21. Further, when $J_{3}=0$, the model provides an example of an 1D spin model which supports a topological quantum phase transition with the critical point at $J_{1}=J_{2} 19$. Moreover, in $d=2$, the model supports a gapless phase for $\left|J_{1}-J_{2}\right| \leq J_{3} \leq J_{1}+J_{2}$ which has a possible connection to a spin liquid state and demonstrates fermion fractionalization at all energy scales $^{22}$. Finally, it has been shown in Ref. 18 that the presence of magnetic field, which induces a gap in the $2 \mathrm{D}$ gapless phase, leads to non-Abelian statistics of the low-lying excitations of the model; these excitations can be viewed as robust qubits in a quantum computer ${ }^{24}$. An extended version of this model has also been suggested in Ref. 25 which has the Hamiltonian

$$
\begin{aligned}
H_{2}=J_{4} & {\left[\sum_{j+l=\text { odd }} \sigma_{j, l}^{y} \sigma_{j+1, l}^{z} \sigma_{j+2, l}^{x}\right.} \\
& \left.+\sum_{j+l=\text { even }} \sigma_{j, l}^{x} \sigma_{j+1, l}^{z} \sigma_{j+2, l}^{y}\right]+H_{1} .
\end{aligned}
$$

The quench dynamics of the 2D Kitaev model has been studied very recently in Ref. 26. It has been shown that for this model, quenching $J_{3}$ takes the system through a 
critical line instead of critical point which leads to unconventional variation of the defect density as a function of the quench rate. In this context, it has also been shown that for a general $d$-dimensional model, where the quench take the system through a $d-m$ dimensional hypersurface characterized by the correlation length exponent $\nu$ and dynamical critical exponent $z$, the defect density obeys $n_{d} \sim 1 / \tau^{m \nu /(z \nu+1)}$. The Kitaev model provides a concrete example of such a quench for $d=2$ and $m=1$. The defect correlation function for such a quench has also been computed in Ref. 26 .

In this work, we extend and elaborate on the results of Ref. 26 and study the quench dynamics of the Kitaev model both in $d=1$ and $d=2$ and the extended Kitaev model in $d=2$. The main results that we have obtained are the following. First, we show that in $1 \mathrm{D}\left(J_{3}=0\right)$, where quenching $J_{1}$ takes the system across the topological quantum critical point located at $J_{1}=J_{2}$, the density of defects produced due to the quench scales as $1 / \sqrt{\tau}$ in the limit of slow quench (large $\tau$ ). We also identify and compute all independent non-zero spin-spin correlation functions and use them to elucidate the spatial extent of the defect correlation function. Second, we outline a general proof of the result reported in Ref. 26 that for a $d$ dimensional quantum model, where the quench take the system through a $d-m$ dimensional hypersurface characterized by the correlation length exponent $\nu$ and dynamical critical exponent $z$, the defect density obeys $n_{d} \sim 1 / \tau^{m \nu /(z \nu+1)}$. Third, we elaborate on the variation of shape and size of the defect correlation function for the 2D Kitaev model with the quench rate and the model parameters. Fourth, we compute the entropy generated due to such a quench and discuss its dependence on the model parameters and the quench rate. Finally, we study the defect scaling law, entropy generation and defect correlation function of the 2D extended Kitaev model described by $\mathrm{H}_{2}$.

The organization of the paper is as follows. In Sec. IIA we analyze the quench dynamics of the Kitaev model in 1D and obtain the quench rate dependence of the defect density. This is followed by Sec. IIB where we compute the 1D correlation functions and use them to discuss the nature of the defect correlation function. Next, in Sec. IIIA we obtain the quench rate dependence of the defect density in 2D. The computation of the defect correlation function is detailed in Sec. IIIB and the entropy generated during the quench process is computed in Sec. IIIC This is followed by the study of quench dynamics of the extended Kitaev model in Sec. IV] Finally, we conclude in Sec. V.

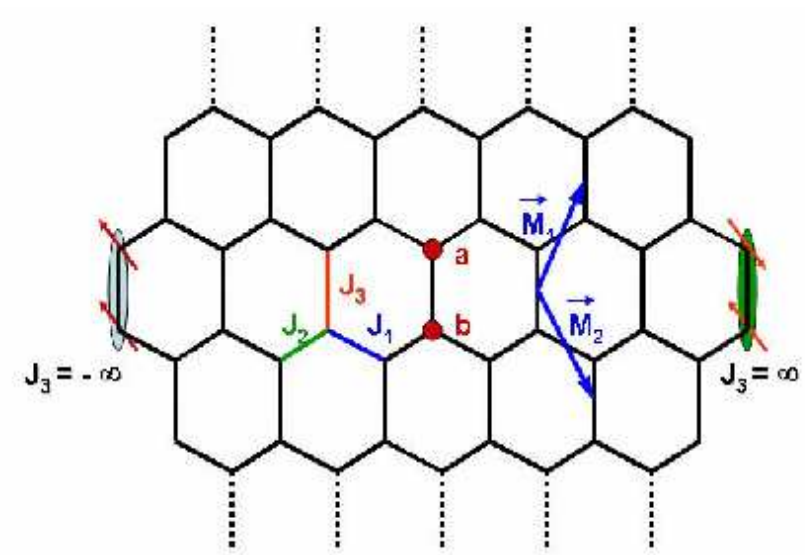

FIG. 1: Schematic representation of the Kitaev model on a honeycomb lattice showings the bonds $J_{1}, J_{2}$ and $J_{3}$. Schematic pictures of the ground states, which correspond to pairs of spins on vertical bonds locked parallel (antiparallel) to each other in the limit of large negative (positive) $J_{3}$, are shown at one bond on the left (right) edge respectively. $\vec{M}_{1}$ and $\vec{M}_{2}$ are spanning vectors of the lattice, and $a$ and $b$ represent inequivalent sites.

\section{QUENCH IN 1D}

\section{A. Defect density}

For $J_{3}=0$, the Kitaev model represents a spin- $1 / 2$ model in 1D with the Hamiltonian

$$
H_{1 \mathrm{D}}=\sum_{n}\left(J_{1} \sigma_{2 n}^{x} \sigma_{2 n+1}^{x}+J_{2} \sigma_{2 n-1}^{y} \sigma_{2 n}^{y}\right)
$$

where $n$ denotes site indices of a one dimensional chain with $N$ sites (we will assume $N$ is a multiple of 4 ). The lattice spacing $a$ and the Planck constant $\hbar$ will be set equal to 1 in the rest of this work. The Hamiltonian in Eq. (3) can be exactly diagonalized using a standard Jordan-Wigner transformation ${ }^{27}$

$$
\begin{aligned}
& a_{n}=\left(\prod_{j=-\infty}^{2 n-1} \sigma_{j}^{z}\right) \sigma_{2 n}^{y}, \\
& b_{n}=\left(\prod_{j=-\infty}^{2 n} \sigma_{j}^{z}\right) \sigma_{2 n+1}^{x},
\end{aligned}
$$

where $a_{n}$ and $b_{n}$ are independent Majorana fermions at site $n$. They satisfy relations such as $a_{n}^{\dagger}=a_{n},\left\{a_{m}, a_{n}\right\}=$ $2 \delta_{m, n}$ and $\left\{a_{m}, b_{n}\right\}=0$. The label $n$ for $a_{n}$ and $b_{n}$ go over $N / 2$ values since that is the number of unit cells. In 
terms of these operators, $H_{1 \mathrm{D}}$ can be written as

$$
\begin{array}{r}
H_{1 \mathrm{D}}=i \sum_{n}\left[J_{1} b_{n} a_{n}+J_{2} b_{n} a_{n+1}\right] \\
=2 i \sum_{k=0}^{\pi}\left[\begin{array}{c}
b_{k}^{\dagger} a_{k}\left(J_{1}+J_{2} e^{i k}\right) \\
\left.+a_{k}^{\dagger} b_{k}\left(-J_{1}-J_{2} e^{-i k}\right)\right],
\end{array}\right.
\end{array}
$$

where the Majorana fermion creation and destruction operators $a_{k}^{\dagger}$ and $a_{k}$ are Fourier components of the $a_{n}$ 's,

$$
a_{n}=\sqrt{\frac{4}{N}} \sum_{k=0}^{\pi}\left[a_{k} e^{i k n}+a_{k}^{\dagger} e^{-i k n}\right]
$$

The sum over $k$ in Eq. (6) only goes over half the Brillouin zone because $a_{n}$ describes a Majorana fermion; the number of modes lying in the range $0 \leq k \leq \pi$ is $N / 4$. [There is a small correction that one has to make in Eq. (6) for the modes with $k=0$ and $\pi$ for which there is no distinction between $k$ and $-k$; these two modes should have a coefficient of $\sqrt{2 / N}$ instead of $\sqrt{4 / N}$. However, we will ignore this correction here because we will be interested in the $N \rightarrow \infty$ limit, and we will change from a sum over $k$ to an integral over $k$.] The operators $a_{k}$ and $a_{k}^{\dagger}$ satisfy the anticommutation relations $\left\{a_{k}, a_{k^{\prime}}^{\dagger}\right\}=\delta_{k k^{\prime}}$ and $\left\{a_{k}, a_{k^{\prime}}\right\}=0$. One can now define a two component fermionic creation operator $\psi_{k}=\left(a_{k} b_{k}\right)$, so that $H_{1 \mathrm{D}}$ can be written as

$$
H_{1 \mathrm{D}}=\sum_{k=0}^{\pi} \psi_{k}^{\dagger} H_{k} \psi_{k},
$$

where $H_{k}=2 i\left(\begin{array}{cc}0 & -J_{1}-J_{2} e^{-i k} \\ J_{1}+J_{2} e^{i k} & 0\end{array}\right)$.

From Eq. (7), we find that $H_{1 \mathrm{D}}$ can be diagonalized leading to an energy spectrum consisting of two bands

$$
E_{k}^{ \pm}= \pm 2 \sqrt{J_{1}^{2}+J_{2}^{2}+2 J_{1} J_{2} \cos k}
$$

Note that the band gap vanishes at $J_{1}= \pm J_{2}$ for $k=\pi$ and 0 respectively, where the bands touch each other. It was shown in Ref. 19 that this vanishing of the energy gap signals a topological phase transition between the two phases of the model at $J_{1}>J_{2}$ and $J_{1}<J_{2}$.

To study the quench of the system across this critical point, we will now consider what happens when we evolve $J_{1}$ linearly in time at a rate $1 / \tau$ from $-\infty$ to $\infty$, keeping $J_{2}$ fixed at some positive value: we take $J_{1}=J_{2} t / \tau$. The ground states of $H_{1 \mathrm{D}}$ in Eq. (7) have $\sigma_{2 n}^{x} \sigma_{2 n+1}^{x}=1$ and -1 for $t=-\infty$ and $\infty$ respectively for all values of $n$. In terms of the Hamiltonian in Eq. (7), the ground and excited states for $J_{1} \rightarrow-\infty$ are respectively given by

$$
\psi_{1 k}=\frac{1}{\sqrt{2}}\left(\begin{array}{c}
1 \\
i
\end{array}\right) \text { and } \psi_{2 k}=\frac{1}{\sqrt{2}}\left(\begin{array}{c}
1 \\
-i
\end{array}\right) .
$$

For $J_{1} \rightarrow \infty$, the ground and excited states are given by $\psi_{2 k}$ and $\psi_{1 k}$ respectively.

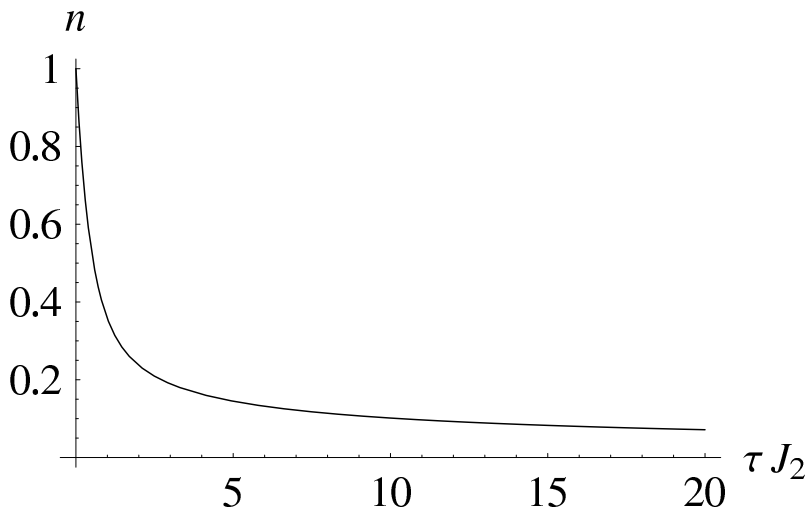

FIG. 2: Defect density produced by quenching $J_{1}$ in $d=1$.

By a change of basis, one can rewrite Eq. (7) in the form $H_{1 \mathrm{D}}=\sum_{k} \psi_{k}^{\prime \dagger} H_{k}^{\prime} \psi_{k}^{\prime}$ where

$$
H_{k}^{\prime}=2\left(\begin{array}{cc}
J_{1}+J_{2} \cos k & -J_{2} \sin k \\
-J_{2} \sin k & -J_{1}-J_{2} \cos k
\end{array}\right) .
$$

Note that unlike Eq. (7), the off-diagonal elements of Eq. (10) do not change with time if $J_{2}$ is held fixed. As a result, the problem of quench dynamics is reduced to solving a standard Landau-Zener problem for each momentum $k^{28}$. The density of defect formation $n$ can thus be found to be ${ }^{29}$

$$
\begin{aligned}
n & =\int_{0}^{\pi} \frac{d k}{\pi} p_{k}, \\
\text { where } p_{k} & =e^{-2 \pi J_{2} \tau \sin ^{2} k}
\end{aligned}
$$

denotes the probability of the system to remain in the initial $\left(J_{1} \rightarrow-\infty\right)$ ground state for momentum $k$. For $J_{2} \tau \gg 1$, the contribution to $n$ comes mainly from the regions near $k=0$ and $\pi$ where $p_{k}=1$. Thus one finds that in the slow quench regime $n \simeq 1 / \sqrt{J_{2} \tau}$. Such a $1 / \sqrt{\tau}$ scaling of defect density conforms to the prediction of Ref. 4. For the present case, it is easy to see from Eq. (8), that the gap $\Delta(k)=E^{+}(k)-E^{-}(k)$ vanishes linearly at the critical point both with the quench parameter $J_{1}$ and with momentum around $k=0$ and $\pi$, so that $z \nu=$ $z=1$. Thus, $n \sim 1 / \tau^{d \nu /(z \nu+1)}=1 / \sqrt{\tau}$ in $1 \mathrm{D}$.

A plot of the defect density as a function of the quench time $\tau$ is shown in Fig. 2. The plot confirms the expected result, that the defect density is maximum for an infinite quench rate $(\tau \rightarrow 0)$, when the system has no time to adjust to the quench and remains in the old ground state leading to a normalized defect density of 1 . As the rate of quench is decreased, $n$ decreases quickly before settling down to a $1 / \sqrt{\tau}$ behavior for large $\tau$.

It is useful to note that the Hamiltonian $H_{k}$ in Eq. (7) can also be written, after a suitable change of basis, as

$$
H_{k}^{\prime}=2\left(\begin{array}{cc}
J_{-} \sin (k / 2) & -i J_{+} \cos (k / 2) \\
i J_{+} \cos (k / 2) & -J_{-} \sin (k / 2)
\end{array}\right),
$$


where $J_{ \pm}=J_{1} \pm J_{2}$. This form is useful if, for instance, one wants to study the effect of quenching $J_{-}$from $-\infty$ to $\infty$ keeping $J_{+}$fixed.

\section{B. Correlation functions}

Let us now consider how the system may be described at the final time $t \rightarrow \infty$ when $J_{1}=\infty$. In principle, the time evolution of the system is unitary, so that it will always be a pure state. However, for each momentum $k$, the wave function is given by $\sqrt{1-p_{k}} \psi_{2 k} e^{-i E_{k}^{2} t}+\sqrt{p_{k}} \psi_{1 k} e^{-i E_{k}^{1} t}$, where $E_{k}^{1,2}= \pm \infty$. As a result, the final density matrix of the system will have off-diagonal terms involving $\psi_{2 k}^{*} \psi_{1 k}$ and $\psi_{1 k}^{*} \psi_{2 k}$ which vary extremely rapidly with time; their effects on physical quantities will therefore average to zero. Hence the final density matrix is effectively diagonal like that of a mixed state 11 , where the diagonal entries are timeindependent as $t \rightarrow \infty$ and are given by $1-p_{k}$ and $p_{k}$. Such a density matrix is associated with an entropy which we will discuss in Sec. IIIC in the context of $2 \mathrm{D}$ Kitaev model.

Using the above density matrix, we will now compute the correlation functions corresponding to the operators $O_{r}=i b_{n} a_{n+r}$, where $r$ is an integer. In terms of the spins, as can be seen from Eq. (44), the operator $O_{r}$ can be written as

$$
\begin{aligned}
O_{0} & =\sigma_{2 n}^{x} \sigma_{2 n+1}^{x}, \quad O_{1}=\sigma_{2 n+1}^{y} \sigma_{2 n+2}^{y}, \\
O_{r} & =\sigma_{2 n+1}^{y}\left(\prod_{j=2 n+2}^{2 n+2 r-1} \sigma_{j}^{z}\right) \sigma_{2 n+2 r}^{y} \text { for } r \geq 2, \\
& =\sigma_{2 n+2 r}^{y}\left(\prod_{j=2 n+2 r+1}^{2 n} \sigma_{j}^{z}\right) \sigma_{2 n+1}^{y} \text { for } r \leq-1 .
\end{aligned}
$$

We will calculate the expectation values of these operators shortly. In principle, one can also consider expectation values of the operators $i a_{n} a_{n+r}$ and $i b_{n} b_{n+r}$; however a direct calculation shows that these vanish if $r \neq 0$. Further, for the Kitaev model, it has been shown that the spin-spin correlations between sites lying on different bonds vanish, i.e., $\left\langle\sigma_{2 n}^{x} \sigma_{2 n+r}^{x}\right\rangle=0$ for $|r| \geq 2^{22}$. Therefore $\left\langle O_{r}\right\rangle$ are the only non-vanishing spin-correlators of the model ${ }^{20}$.

To compute $\left\langle O_{r}\right\rangle$ we note that $O_{r}$ can be expressed in terms of the fermion operators $a_{k}$ and $b_{k}$. This will in general involve summations over two different momenta $k$ and $k^{\prime}$. However, when $\left\langle O_{r}\right\rangle$ is computed in a direct product of states involving $a_{k}$ and $b_{k}$, only terms in which $k^{\prime}=k$ will contribute. In the limit $N \rightarrow \infty$, the relevant part of $O_{r}$ which contributes to the correlation function can be written as

$$
O_{r}=-\frac{4 i}{N} \sum_{k=0}^{\pi}\left[b_{k}^{\dagger} a_{k} e^{i k r}-a_{k}^{\dagger} b_{k} e^{i k r}\right]
$$

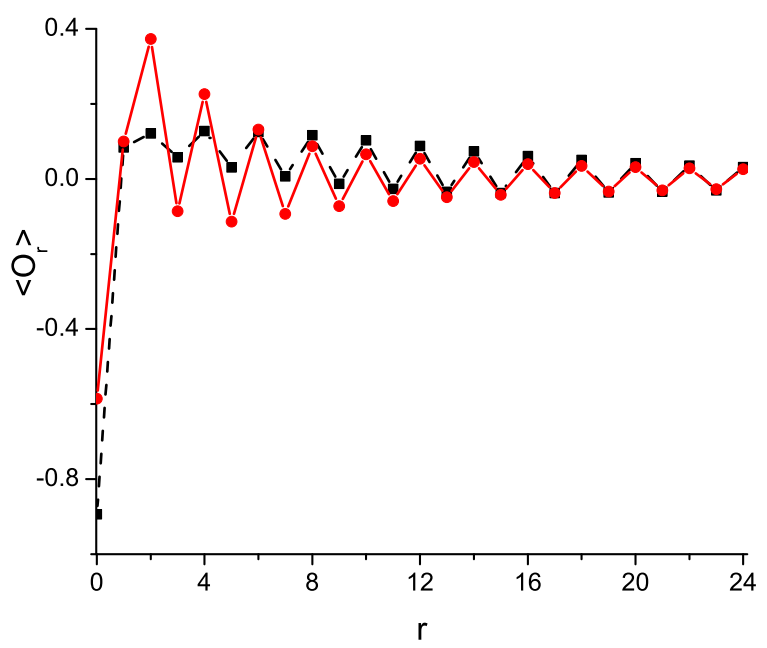

FIG. 3: Plot of correlation function $\left\langle O_{r}\right\rangle$ as a function of $r$ for $J_{2} \tau=10$ (red circles and red solid line) and $J_{2} \tau=1$ (black squares and black dashed line). $\left\langle O_{r}\right\rangle$ shows a damped oscillatory behavior as a function of $r$.

Using the wave functions given in Eq. (9), we find that

$$
\left\langle O_{r}\right\rangle= \pm \int_{0}^{\pi} d k \cos (k r)= \pm \delta_{r, 0}
$$

where the + and - signs refer to the ground states of $J_{1}=-\infty$ and $\infty$ respectively. This is expected since $\sigma_{2 n}^{x} \sigma_{2 n+1}^{x}= \pm 1$ while all other correlations vanish in those two states. Finally, after quench, in a state in which we have a mixture of the ground and excited states of $J_{1}=\infty$ with probabilities $1-p_{k}$ and $p_{k}$ respectively, we find that

$$
\left\langle O_{r}\right\rangle=-\delta_{r, 0}+\frac{2}{\pi} \int_{0}^{\pi} d k p_{k} \cos (k r)
$$

A plot of $\left\langle O_{r}\right\rangle$ as a function of $r$ is shown for representative values of $J_{2} \tau=1,10$ in Fig. 3. We find that $\left\langle O_{r}\right\rangle$ shows a damped oscillatory behavior. Note that since $\left\langle O_{r}\right\rangle=-\delta_{r, 0}$ for the ground state of $H_{1 \mathrm{D}}$ for $J_{1} \rightarrow \infty$, the plot of $\left\langle O_{r}\right\rangle$ in the state of the system after the quench provides a direct measurement of the spatial extent of the correlation between the defects generated during the quench.

For $J_{2} \tau \gg 1$, the dominant contribution in the integral in Eq. (16) comes from the regions near $k=0$ and $\pi$ as can be seen from the expression for $p_{k}$ in Eq. (11). One can combine these two regions, and write the expression 
in (16) approximately as

$$
\begin{aligned}
\left\langle O_{r}\right\rangle=-\delta_{r, 0}+\frac{2}{\pi} \int_{0}^{\infty} d k e^{-2 \pi J_{2} \tau k^{2}} & \times[\cos (k r)+\cos \{(\pi-k) r\}] \\
= & -\delta_{r, 0}+\frac{1+(-1)^{r}}{\pi} \frac{e^{-r^{2} /\left(8 \pi J_{2} \tau\right)}}{\sqrt{2 J_{2} \tau}} .
\end{aligned}
$$

Note that this vanishes if $r$ is odd. For a given value of $J_{2} \tau$, the expression in Eq. (17) decreases with increasing $r$, particularly for $r>\sqrt{8 \pi J_{2} \tau}$. On the other hand, for a given large value of $r$, Eq. (17) has a maximum at $\tau=r^{2} /\left(4 \pi J_{2}\right)$. The fact that the crossover in both cases occurs around $r \sim \sqrt{4 \pi J_{2} \tau}$ signals the fact that the associated length scale for the defect correlation function is of order $\sqrt{4 \pi J_{2} \tau}$.

\section{Sum rule}

There is a sum rule that we can write down for $\left\langle O_{r}\right\rangle$. From Eq. (16), we see that

$$
O_{\text {total }} \equiv \sum_{r=-\infty}^{\infty}\left\langle O_{r}\right\rangle=-1+2 p_{0}
$$

where we have used the identity $\sum_{r} e^{i k r}=2 \pi \delta(k)$ for $-\pi<k<\pi$. Going back to Eq. (10), we see that for $k=0$, the Hamiltonians at different times commute with each other irrespective of how $J_{1}$ is varied in time from $-\infty$ to $\infty$. This means that if we start with the ground state of $J_{1}=-\infty$, no transition will occur at any time, and we will have $p_{0}=1$. Eq. (18) then implies that $O_{\text {total }}=1$.

\section{2D KITAEV MODEL}

\section{A. Defect density}

When $J_{3} \neq 0$, the Kitaev model with Hamiltonian given by Eq. (1) describes a spin model on a hexagonal 2D lattice. Usually spin models are not exactly solvable in two dimensions. One of the main properties of the Kitaev model which makes it theoretically attractive is that, even in $2 \mathrm{D}$, it can be mapped onto a non-interacting fermionic model by a suitable JordanWigner transformation $18,19,20,21$. In terms of the Majorana fermions $a_{j l}$ and $b_{j l}$ one can write

$$
\begin{aligned}
& a_{j l}=\left(\prod_{i=-\infty}^{j-1} \sigma_{i l}^{z}\right) \sigma_{j l}^{y} \text { for even } j+l, \\
& b_{j l}=\left(\prod_{i=-\infty}^{j-1} \sigma_{i l}^{z}\right) \sigma_{j l}^{x} \text { for odd } j+l .
\end{aligned}
$$

Such a transformation maps the spin Hamiltonian $H$ in Eq. (1) to a fermionic Hamiltonian given by

$$
\begin{aligned}
H_{2 \mathrm{D}}=i \sum_{\vec{n}}[ & J_{1} b_{\vec{n}} a_{\vec{n}-\vec{M}_{1}}+J_{2} b_{\vec{n}} a_{\vec{n}+\vec{M}_{2}} \\
& \left.+J_{3} D_{\vec{n}} b_{\vec{n}} a_{\vec{n}}\right],
\end{aligned}
$$

where $\vec{n}=\sqrt{3} \hat{i} n_{1}+\left(\frac{\sqrt{3}}{2} \hat{i}+\frac{3}{2} \hat{j}\right) n_{2}$ denote the midpoints of the vertical bonds. Here $n_{1}, n_{2}$ run over all integers so that the vectors $\vec{n}$ form a triangular lattice whose vertices lie at the centers of the vertical bonds of the underlying honeycomb lattice; the Majorana fermions $a_{\vec{n}}$ and $b_{\vec{n}}$ sit at the top and bottom sites respectively of the bond labeled $\vec{n}$. The vectors $\vec{M}_{1}=\frac{\sqrt{3}}{2} \hat{i}+\frac{3}{2} \hat{j}$ and $\vec{M}_{2}=\frac{\sqrt{3}}{2} \hat{i}-\frac{3}{2} \hat{j}$ are spanning vectors for the reciprocal lattice, and $D_{\vec{n}}$ can take the values \pm 1 independently for each $\vec{n}$. The crucial point that makes the solution of $\mathrm{Ki}-$ taev model feasible is that $D_{\vec{n}}$ commutes with $H_{2 \mathrm{D}}$, so that all the eigenstates of $H_{2}$ D can be labeled by specific values of $D_{\vec{n}}$. It has been shown that for any value of the parameters $J_{i}$, the ground state of the model always corresponds to $D_{\vec{n}}=1$ on all the bonds. Since $D_{\vec{n}}$ is a constant of motion, the dynamics of the model starting from any ground state never takes the system outside the manifold of states with $D_{\vec{n}}=1$.

For $D_{\vec{n}}=1$, it is straightforward to diagonalize $H_{2 \mathrm{D}}$ in momentum space. We define Fourier transforms of the Majorana operators $a_{\vec{n}}$ as

$$
a_{\vec{n}}=\sqrt{\frac{4}{N}} \sum_{\vec{k}}\left[a_{\vec{k}} e^{i \vec{k} \cdot \vec{n}}+a_{\vec{k}}^{\dagger} e^{-i \vec{k} \cdot \vec{n}}\right]
$$

(and similarly for $b_{\vec{n}}$ ), where $N$ is the number of sites (assumed to be even, so that the number of unit cells $N / 2$ is an integer), and the sum over $\vec{k}$ extends over half the Brillouin zone of the $2 \mathrm{D}$ hexagonal lattice. We then obtain $H_{2 \mathrm{D}}=\sum_{\vec{k}} \psi_{\vec{k}}^{\dagger} H_{\vec{k}} \psi_{\vec{k}}$, where $\psi_{\vec{k}}^{\dagger}=\left(a_{\vec{k}}^{\dagger}, b_{\vec{k}}^{\dagger}\right)$, and $H_{\vec{k}}$ can be expressed in terms of Pauli matrices $\sigma^{1,2,3}$ as

$$
\begin{aligned}
H_{\vec{k}}= & 2\left[J_{1} \sin \left(\vec{k} \cdot \vec{M}_{1}\right)-J_{2} \sin \left(\vec{k} \cdot \vec{M}_{2}\right)\right] \sigma^{1} \\
& +2\left[J_{3}+J_{1} \cos \left(\vec{k} \cdot \vec{M}_{1}\right)+J_{2} \cos \left(\vec{k} \cdot \vec{M}_{2}\right)\right] \sigma^{2} .
\end{aligned}
$$

The energy spectrum of $H_{2 \mathrm{D}}$ therefore consists of two bands with energies

$$
\begin{aligned}
E_{\vec{k}}^{ \pm}= \pm 2 & {\left[\left(J_{1} \sin \left(\vec{k} \cdot \vec{M}_{1}\right)-J_{2} \sin \left(\vec{k} \cdot \vec{M}_{2}\right)\right)^{2}\right.} \\
& \left.+\left(J_{3}+J_{1} \cos \left(\vec{k} \cdot \vec{M}_{1}\right)+J_{2} \cos \left(\vec{k} \cdot \vec{M}_{2}\right)\right)^{2}\right]^{1 / 2} .
\end{aligned}
$$

We note for $\left|J_{1}-J_{2}\right| \leq J_{3} \leq\left(J_{1}+J_{2}\right)$, these bands touch each other so that the energy gap $\Delta_{\vec{k}}=E_{\vec{k}}^{+}-E_{\vec{k}}^{-}$vanishes for special values of $\vec{k}$ leading to the gapless phase of the model $18,19,20,25$.

We will now quench $J_{3}(t)=J t / \tau$ at a fixed rate $1 / \tau$, from $-\infty$ to $\infty$, keeping $J, J_{1}$ and $J_{2}$ fixed at some nonzero values; we have introduced the quantity $J$ to fix the 
scale of energy. We note that the ground states of $H_{2 \mathrm{D}}$ corresponding to $J_{3} \rightarrow-\infty(\infty)$ are gapped and have $\sigma_{j, l}^{z} \sigma_{j, l+1}^{z}=1(-1)$ for all lattice sites $(j, l)$. To study the state of the system after the quench, we first note that after an unitary transformation $U=\exp \left(-i \sigma_{1} \pi / 4\right)$, one can write $H_{2 \mathrm{D}}=\sum_{\vec{k}} \psi_{\vec{k}}^{\prime \dagger} H_{\vec{k}}^{\prime} \psi_{\vec{k}}^{\prime}$, where $H_{\vec{k}}^{\prime}=U H_{\vec{k}} U^{\dagger}$ is given by

$$
\begin{aligned}
H_{\vec{k}}^{\prime}= & 2\left[J_{1} \sin \left(\vec{k} \cdot \vec{M}_{1}\right)-J_{2} \sin \left(\vec{k} \cdot \vec{M}_{2}\right)\right] \sigma^{1} \\
& +2\left[J_{3}(t)+J_{1} \cos \left(\vec{k} \cdot \vec{M}_{1}\right)+J_{2} \cos \left(\vec{k} \cdot \vec{M}_{2}\right)\right] \sigma^{3} .
\end{aligned}
$$

Hence the off-diagonal elements of $H_{\vec{k}}^{\prime}$ remain time independent and the problem of quench dynamics reduces to a Landau-Zener problem for each $\vec{k}$. The defect density can then be computed following a standard prescription 28

$$
\begin{aligned}
n & =\frac{1}{A} \int_{\vec{k}} d^{2} \vec{k} p_{\vec{k}}, \\
p_{\vec{k}} & =e^{-2 \pi \tau\left[J_{1} \sin \left(\vec{k} \cdot \vec{M}_{1}\right)-J_{2} \sin \left(\vec{k} \cdot \vec{M}_{2}\right)\right]^{2} / J},
\end{aligned}
$$

where $A=4 \pi^{2} /(3 \sqrt{3})$ denotes the area of half the Brillouin zone over which the integration is carried out. Since the integrand in Eq. (25) is an even function of $\vec{k}$, one can extend the region of integration over the full Brillouin zone. This region can be chosen to be a rhombus with vertices lying at $\left(k_{x}, k_{y}\right)=( \pm 2 \pi / \sqrt{3}, 0)$ and $(0, \pm 2 \pi / 3)$. Introducing two independent integration variable $v_{1}, v_{2}$, each with a range $0 \leq v_{1}, v_{2} \leq 1$, one finds that

$$
k_{x}=2 \pi \frac{v_{1}+v_{2}-1}{\sqrt{3}}, \quad k_{y}=2 \pi \frac{v_{2}-v_{1}}{3} .
$$

Such a substitution covers the rhombus uniformly and facilitates the numerical integration necessary for computing $n$.

A plot of $n$ as a function of the quench time $J \tau$ and $\alpha=$ $\tan ^{-1}\left(J_{2} / J_{1}\right)$ (we have taken $J_{1[2]}=J \cos (\alpha)[\sin (\alpha)]$ ) is shown in Fig. 4. We note that the density of defects produced is maximum when $J_{1}=J_{2}$. This is due to the fact that the length of the gapless line through which the system passes during the quench is maximum at this point. This allows the system to remain in the non-adiabatic state for the maximum time during the quench, leading to the maximum density of defects. For $J_{1} / J_{3}>2 J_{2} / J_{3}$, the system does not pass through a gapless phase during the quench, and the defect production is exponentially suppressed.

For sufficiently slow quench $2 \pi J \tau \gg 1, p_{\vec{k}}$ is exponentially small for all values of $\vec{k}$ except in the region near the line

$$
J_{1} \sin \left(\vec{k} \cdot \vec{M}_{1}\right)-J_{2} \sin \left(\vec{k} \cdot \vec{M}_{2}\right)=0,
$$

and the contribution to the momentum integral in (25) comes from values of $\vec{k}$ close to this line of zeroes. We note that the line of zeroes where $p_{\vec{k}}=1$ precisely corresponds to the zeroes of the energy gap $\Delta_{\vec{k}}$ as $J_{3}$ is varied

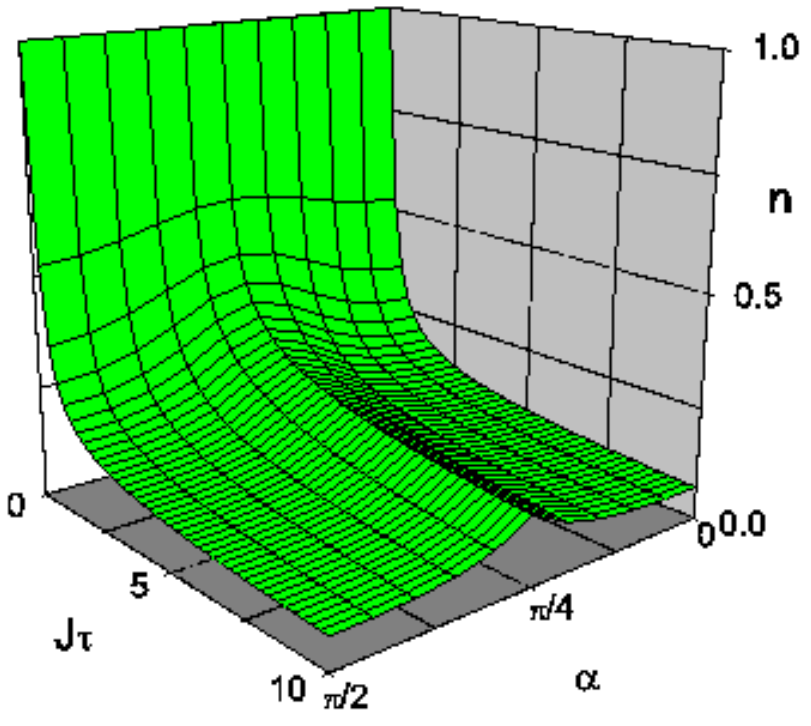

FIG. 4: Plot of defect density $n$ as a function of the quench time $J \tau$ and $\alpha=\tan ^{-1}\left(J_{2} / J_{1}\right)$. The density of defects is maximum at $J_{1}=J_{2}$.

for a fixed $J_{2}$ and $J_{1}$. Thus the system becomes nonadiabatic when it passes through the intermediate gapless phase in the interval $\left|J_{1}-J_{2}\right| \leq J_{3}(t) \leq\left(J_{1}+J_{2}\right)$. It is then easy to see, by expanding $p_{\vec{k}}$ about this line that in the limit of slow quench, the defect density scales as $n \sim 1 / \sqrt{\tau}$. We note that the scaling of the defect density with the quench rate in a quench where the system passes through a critical line in momentum space is different from the situation where the quench takes the system through a critical point. In the latter case, for the Kitaev model which has $z=\nu=1$, Ref. 4 predicts a defect density $n \sim 1 / \tau$ for $d=2$. Thus the defect density crucially depends on the dimensionality of the critical surface through which the system passes during the quench. This observation leads to a simple but general conclusion which we present below.

Consider a $d$-dimensional model with $z=\nu=1$ described by a Hamiltonian

$$
H_{d}=\sum_{\vec{k}} \psi_{\vec{k}}^{\dagger}\left(\begin{array}{cc}
\epsilon(\vec{k}, t) & \Delta(\vec{k}) \\
\Delta^{*}(\vec{k}) & -\epsilon(\vec{k}, t)
\end{array}\right) \psi_{\vec{k}},
$$

where $\epsilon(\vec{k}, t)=\epsilon(\vec{k}) t / \tau$. Now let us assume that the quench takes such a system through a critical surface of $d-m$ dimensions. The defect density for a sufficiently slow quench can be expressed $a^{28,29}$

$$
\begin{aligned}
n & =\frac{1}{A} \int_{\mathrm{BZ}} d^{d} k p(\vec{k}), \text { where } p(\vec{k})=e^{-\pi \tau f(\vec{k})}, \\
& \simeq \frac{1}{A} \int_{\mathrm{BZ}} d^{d} k \exp \left[-\tau \sum_{\alpha \beta=1, m} g_{\alpha \beta} k_{\alpha} k_{\beta}\right] \\
& \sim 1 / \tau^{m / 2}
\end{aligned}
$$

where $p_{\vec{k}}$ is the defect probability for momentum $\vec{k}$, 
$f(\vec{k})=|\Delta(\vec{k})|^{2} /|\epsilon(\vec{k})|$ vanishes on the $d-m$ dimensional critical surface, $\alpha, \beta$ denote one of the $m$ orthogonal directions to the critical surface and $g_{\alpha \beta}=$ $\left(\partial^{2} f(\vec{k}) / \partial k_{\alpha} \partial k_{\beta}\right)_{\vec{k} \in \text { critical surface. We note that this re- }}$ sult depends only on the property that $f(\vec{k})$ has to vanish on a $d-m$ dimensional surface, and not on the precise nature of $f(\vec{k})$. For $m=d$, where the quench takes the system through a critical point, our results coincide with that of Ref. 4.

Finally we generalize our arguments for models where the $d-m$ dimensional hypersurface is characterized by correlation length exponent $\nu$ and dynamical critical exponent $z$. Let us assume that the system is described by a Hamiltonian $H[\lambda(t)]$ with quasi-energy eigenvalues $E(\vec{k}, t)$ and that the time evolution of the parameter $\lambda(t)=\lambda_{0}(t / \tau)$ takes the system through the critical point $\lambda_{0}=\lambda_{c}$ at $t=t_{0}$. First we note that for large $\tau$, a non-vanishing probability of defect formation requires the non-adiabaticity condition $|\Delta(\vec{k})|^{2} \sim|\partial E(\vec{k}, t) / \partial t|$ [4]. Also, since $\partial E(\vec{k}, t) / \partial t=(\partial E(\vec{k}, t) / \partial \lambda) \tau^{-1}$ and near the critical point $E \sim \lambda^{z \nu}$, we get

$$
\Delta^{2} \sim \tau^{-1} \lambda^{z \nu-1}
$$

Further, as shown in Ref. 4, near any point on the critical surface, quite generally, one has $\Delta \sim|k|^{z}, \lambda \sim k^{1 / \nu}$ and $k \sim 1 / \tau^{\nu /(z \nu+1)}$. Using these relations we find from Eq. 30 that on any point near the gapless surface

$$
\Delta \sim 1 / \tau^{z \nu /(z \nu+1)}
$$

Next, let us consider the available phase space for formation of defects. When the quench takes the system through a $d-m$ dimensional hypersurface in momentum space, the available phase space is $\Omega \sim k^{m} \sim \Delta^{m / z}$. Since this available phase space is directly proportional to the defect density $\stackrel{4}{\underline{4}}$, we find, using Eq. (31),

$$
n \sim \Omega \sim \Delta^{m / z} \sim 1 / \tau^{m \nu /(z \nu+1)}
$$

This generalizes the scaling law for defect density to arbitrary critical systems. Note that for $z=\nu=1$, we recover our earlier result $n \sim 1 / \tau^{m / 2}$ (Eq. (29)). For $m=d$, which represents quench through a critical point, we also recover the result of Ref. $4\left(n \sim 1 / \tau^{d \nu /(z \nu+1)}\right)$ as a special case.

\section{B. Defect Correlation}

The calculation of the correlation function can be accomplished along similar lines as in 1D. First, we define the operators

$$
O_{\vec{r}}^{2 \mathrm{D}}=i b_{\vec{n}} a_{\vec{n}+\vec{r}} .
$$

In terms of the spin operators, we have $O_{\overrightarrow{0}}^{2 \mathrm{D}}=\sigma_{j, l}^{z} \sigma_{j, l+1}^{z}$. For $\vec{r} \neq \overrightarrow{0}, O_{\vec{r}}^{2 \mathrm{D}}$ can be written as a product of spin operators going from a $b$ site at $\vec{n}=(j, l)$ to an $a$ site at $\vec{n}+\vec{r}=\left(j^{\prime}, l^{\prime}\right)$ : the product will begin with a $\sigma^{x}$ or $\sigma^{y}$ and end with a $\sigma^{x}$ or $\sigma^{y}$ with a string of $\sigma^{z}$ 's in between, where the choice of the initial and final $\sigma$ matrices depends on whether the values of $j+l$ and $j^{\prime}+l^{\prime}$ are even or odd. Note that $O_{\vec{r}}^{2 \mathrm{D}}$ for $\vec{r} \neq \overrightarrow{0}$ measures correlation between the defects produced during the quench. In particular, a plot of the correlation function $\left\langle O_{\vec{r}}^{2 \mathrm{D}}\right\rangle$ versus $\vec{r}$ in the defect ground state provides an estimate of the shape and spatial extent of the defect correlations produced during the quench. Note that $\left(O_{\vec{r}}^{2 \mathrm{D}}\right)^{2}=1$, so that all the moments of $O_{\vec{r}}^{2 \mathrm{D}}$ can be found trivially: $\left\langle\left(O_{\vec{r}}^{2 \mathrm{D}}\right)^{n}\right\rangle=\left\langle O_{\vec{r}}^{2 \mathrm{D}}\right\rangle$ if $n$ is odd and $=1$ if $n$ is even.

$O_{\vec{r}}^{2 D}$ can be written in terms of the Majorana fermion operators $a_{\vec{k}}$ and $b_{\vec{k}}$; this again involves a sum over two different momenta $\vec{k}$ and $\vec{k}^{\prime}$. However, the expectation value of $O_{\vec{r}}^{2 D}$ in a direct product of states involving $\vec{k}$ only gets a contribution from terms in which $\vec{k}^{\prime}=\vec{k}$. It turns out that the relevant part of $O_{\vec{r}}^{2 D}$ contributes to the expectation values can be written as,

$$
O_{\vec{r}}^{2 D}=\frac{4 i}{N} \sum_{\vec{k}}\left[b_{\vec{k}}^{\dagger} a_{\vec{k}} e^{i \vec{k} \cdot \vec{r}}-a_{\vec{k}}^{\dagger} b_{\vec{k}} e^{-i \vec{k} \cdot \vec{r}}\right]
$$

The ground state and excited states for $J_{3}=-\infty$ are given by $\psi_{1 \vec{k}}$ and $\psi_{2 \vec{k}}$ respectively, while the two states are interchanged for $J_{3}=\infty$. Using Eq. (9), we find that

$$
\left\langle O_{\vec{r}}^{2 \mathrm{D}}\right\rangle= \pm \frac{4}{N} \sum_{\vec{k}} \cos (\vec{k} \cdot \vec{r})
$$

where the + and - signs refer to the ground states of $J_{3}=-\infty$ and $\infty$ respectively. This confirms our earlier expectation that in the ground states of $J_{3} \rightarrow-\infty(\infty)$, $\left\langle O_{\vec{r}}^{2 \mathrm{D}}\right\rangle= \pm \delta_{\vec{r}, \overrightarrow{0}}$. Finally, in the state after quench, in which we have a mixture of the ground and excited states of $J_{3}=\infty$ with probabilities $1-p_{\vec{k}}$ and $p_{\vec{k}}$ respectively, we find that

$$
\left\langle O_{\vec{r}}^{2 \mathrm{D}}\right\rangle=-\delta_{\vec{r}, \overrightarrow{0}}+\frac{2}{A} \int d^{2} \vec{k} p_{\vec{k}} \cos (\vec{k} \cdot \vec{r}),
$$

where the integral over momentum runs over half the Brillouin zone with area $A$. Note that the full Brillouin zone as well as $p_{\vec{k}}$ remains invariant under a reflection through the point $\vec{k}=(\pi / \sqrt{3}, 0): k_{x} \rightarrow 2 \pi / \sqrt{3}-k_{x}$, $k_{y} \rightarrow-k_{y}$. However, $\cos (\vec{k} \cdot \vec{r})$ changes by a factor of $(-1)^{n_{2}}$, if the components of $\vec{r}$ are given by $x=\sqrt{3}\left(n_{1}+\right.$ $\left.n_{2} / 2\right)$ and $y=3 n_{2} / 2$. Hence, $\left\langle O_{\vec{r}}^{2 \mathrm{D}}\right\rangle=0$ for odd values of $n_{2}$.

For large values of $\tau$, substituting the expression in Eq. (25) in the above integral, we find that the dominant contribution comes from the region near the line given in Eq. (27). Thus at every point $\vec{k}_{0}$ lying on that line, we can introduce variables $k_{\|}$and $k_{\perp}$ which vary along the line and perpendicular to it along the directions $\hat{n}_{\|}$and $\hat{n}_{\perp}$ respectively. Close to $\vec{k}_{0}$, the integrand in Eq. (36) will take the form $\exp \left[-a \tau k_{\perp}^{2} \pm i\left(\vec{k}_{0}+k_{\|} \hat{n}_{\|}+k_{\perp} \hat{n}_{\perp}\right) \cdot \vec{r}\right]$, 


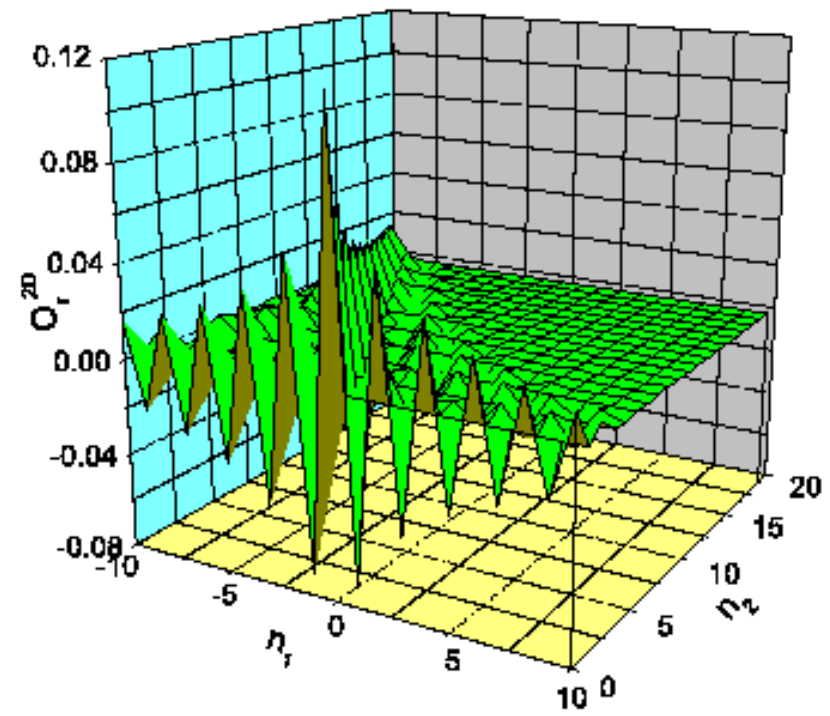

FIG. 5: Plot of $O_{\vec{r}}^{2 \mathrm{D}}$ sans the delta function peak at the origin for $J_{1}=J_{2}=J$ and $J \tau=10$ as a function of $n_{1}$ and $n_{2}$ (see text for details). The spatial anisotropy of the defect correlation function is clearly evident even for $J_{1}=J_{2}$.

where $a$ is a number of order 1 whose value depends on $\vec{k}_{0}$. The integral over $k_{\perp}$ will give a factor of $\exp \left[-\left(\vec{r} \cdot \hat{n}_{\perp}\right)^{2} /(4 a \tau)\right] / \sqrt{a \tau}$. Thus we find that the density of defects is of order $1 / \sqrt{\tau}$ in accordance with Eq. (29). This also leads us to expect that the spatial range of the defect correlation should go as $\sqrt{\tau}$.

Next we consider the shape of the defect correlation function. For this purpose, we evaluate Eq. (36) numerically so as to obtain the $\vec{r}$ dependence of $\left\langle O_{\vec{r}}^{2 \mathrm{D}}\right\rangle$. In general, we expect the correlation will be anisotropic in space if $J_{1} / J_{2} \gg 1$ or $\ll 1$ which can be most easily seen from the fact that setting $J_{1}=0$ or $J_{2}=0$ leads to the 1D result derived in Sec. IIB A plot of the correlation function $\left\langle O_{\vec{r}}^{2 \mathrm{D}}\right\rangle$, without the delta function peak at $\vec{r}=0$, and as a function of $n_{1}$ and $n_{2}$, where $x=\sqrt{3}\left(n_{1}+n_{2} / 2\right)$ and $y=3 n_{2} / 2$ is shown in Fig. 5. In this plot, we have omitted the delta function contribution to $\left\langle O_{\vec{r}=0}^{2 \mathrm{D}}\right\rangle$ in order to make the correlations at $\vec{r} \neq \overrightarrow{0}$ visible. In the $x$ direction, the correlations oscillate; the amplitude of oscillations decays monotonically with $x$, in a qualitatively similar manner to the $1 \mathrm{D}$ correlation function $O_{r}$ shown in Fig. 2 for $y=n_{2}=0$. The correlations decay in a monotonic way with $y$ for $x=n_{1}+n_{2} / 2=0$ (along the straight line at an angle $\theta=\tan ^{-1}(-0.5)$ in the figure). Thus the correlations behave quite anisotropically even for $J_{1}=J_{2}$.

We now aim at obtaining an understanding of the variation of the spatial dependence of $\left\langle O_{r}^{2 \mathrm{~d}}\right\rangle$ with the parameters $J_{1}$ and $J_{2}$. Such a variation can be analytically understood by noting that for $J \tau \gg 1$, the maximum contribution to $\left\langle O_{\vec{r}}\right\rangle$ comes from around the wave vector $\vec{k}_{0}$ for which $p\left(\vec{k}_{0}\right)=1$. For $J_{2} \gg(\ll) 1$, this occurs when $\sin \left[\vec{k} \cdot \vec{M}_{2}\left(\vec{M}_{1}\right)\right]=0$ which yields $\vec{k}_{0}=\pi(\sqrt{3} \hat{i} \mp \hat{j}) / 2$. The
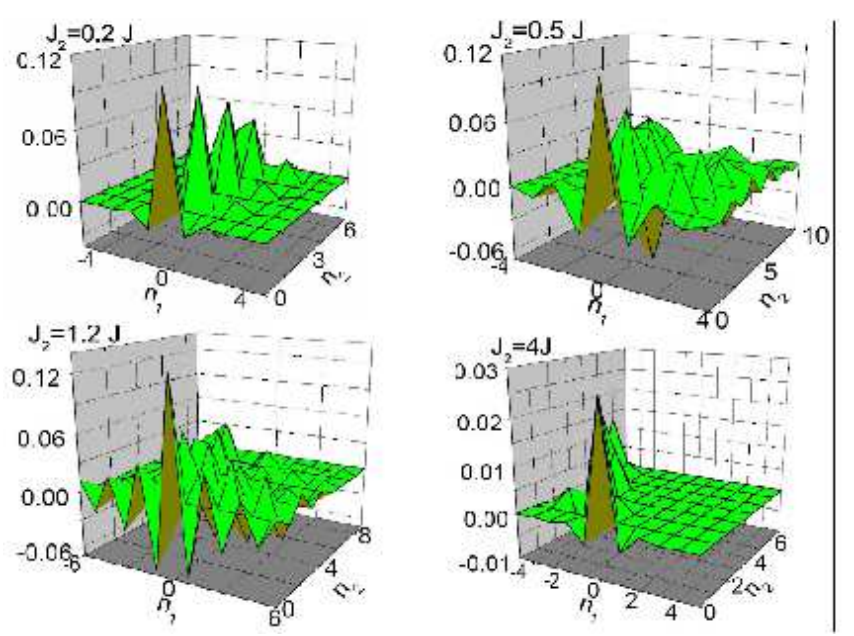

FIG. 6: Plot of $\left\langle O_{r}^{2 \mathrm{~d}}\right\rangle$ sans the delta function peak at the origin as a function of $\vec{r}$ for several representative values of $J_{2} / J$ for $J_{1}=J$ and $J \tau=5$. The plot displays the change in the shape of defect correlation function as a function of $J_{2} / J_{1}$ (see text for details).

maximum contribution to $\left\langle O_{\vec{r}}^{2 \mathrm{D}}\right\rangle$ occurs where $\cos \left(\vec{k}_{0} \cdot \vec{n}\right)$ is maximum, ie., $\vec{k}_{0} \cdot \vec{n}=0$. Thus for $J_{2} \gg(\ll) J_{1},\left\langle O_{\vec{r}}^{2 \mathrm{D}}\right\rangle$ is expected to be maximal along the lines $n_{1}+n_{2}=$ $0\left(n_{2}=0\right)$ in the $n_{1}-n_{2}$ plane. This expectation is confirmed as seen in Fig. [6] which shows $\left\langle O_{r}^{2 \mathrm{~d}}\right\rangle$ for several representative values of $J_{2} / J$ for a fixed $J_{1}=J$ and $J \tau=5$. We find that $\left\langle O_{\vec{r}}\right\rangle$ is maximal along $n_{2}=0\left(n_{1}+n_{2}=0\right)$ line for $J_{2}=5(0.25) J_{1}$. This clearly shows that the defects produced in the quench will be highly anisotropic in this limits. For intermediate values of $J_{1}$ and $J_{2}$, the anisotropy in $\left\langle O_{r}^{2 \mathrm{~d}}\right\rangle$ can be similarly deduced by first finding $\vec{k}_{0}$ for which $p_{\vec{k}_{0}}=0$ and then computing $\vec{n}$ for which $\vec{k}_{0} \cdot \vec{n}$ vanishes. The gradual evolution of the shape of $\left\langle O_{r}^{2 \mathrm{~d}}\right\rangle$ as we go from the limit $J_{2} \ll J_{1}$ to the limit $J_{2} \ll J_{1}$ can be seen from in Fig. 6.

To obtain a more detailed picture of the spatial anisotropy of the defect correlations as a function of $J_{1} / J_{2}$ we define a parameter $\alpha: J_{1[2]}=J \cos (\alpha)[\sin (\alpha)]$. A variation of $\alpha$ therefore changes the ratio $J_{1} / J_{2}$ from 0 to $\infty$ while fixing $J_{1}^{2}+J_{2}^{2}=J^{2}=1$. The plot of $\left\langle O_{\vec{r}}^{2 \mathrm{~d}}\right\rangle$ at points $\left(n_{1}, n_{2}\right)=(-1,0)$ (on the $x$ axis of the $n_{1}-n_{2}$ plane), $\left(n_{1}, n_{2}\right)=(2,-2)$ (along the $-45^{\circ}$ line in the $n_{1}-n_{2}$ plane) and $\left(n_{1}, n_{2}\right)=(0,-2)$ (on the $y$ axis of the $n_{1}-n_{2}$ plane) as a function of $\alpha$ shown in Fig. 7 clearly reveal the nature of the anisotropy of the correlation function. We find that as the ratio of $J_{1} / J_{2}=\tan (\alpha)$ is varied from 0 to $\infty$, the correlation on the representative point $(1,0)$ along the $x$ axis increases till it reaches the point $J_{1}=J_{2}(\alpha=\pi / 4)$ and then decays to 0 as $\alpha$ approaches $\pi / 2$. This signifies that the correlation along the $x$ axis in the $n_{1}-n_{2}$ plane becomes maximum for $J_{1}=J_{2}$. On the other hand, for the representative point $(0,2)$ on the $y$ axis and $2,-2$ along the line with slope $-45^{\circ}$, the correlation becomes maximum when $J_{2} \ll J_{1}$ 


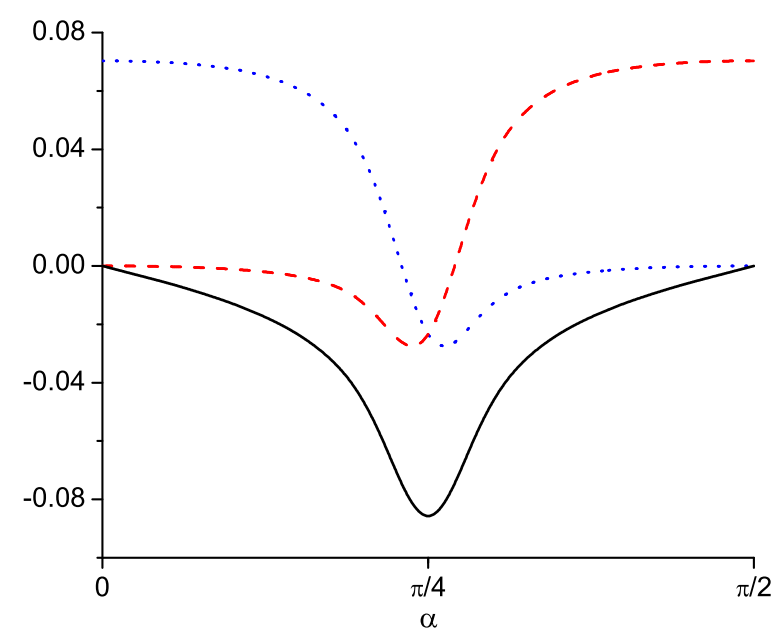

FIG. 7: Plot of $\left\langle O_{r}^{2 \mathrm{~d}}\right\rangle$ (sans the delta function peak) at representative points $(-1,0)$ on the $x$ axis (black solid line) $(0,2)$ on the $y$ axis (blue dotted line) and $(2,-2)$ along $-45^{\circ}$ in the $n_{1}-n_{2}$ plane (red dashed line) as a function of $\alpha=\tan ^{-1}\left(J_{2} / J_{1}\right)$ for fixed $J^{2}=1$.

$(\alpha=0)$ and $J_{2} \gg J_{1}(\alpha=\pi / 2)$ respectively, as expected from Fig. 6. This lead us to conclude that the spatial anisotropy of the defect correlation function $\left\langle O_{\vec{r}}^{2 d}\right\rangle$ depends crucially on the ratio of $J_{1} / J_{2}$.

Finally we note that we can obtain a measure of the spatial extent of the defect correlation function by calculating

$$
\left\langle\vec{r}^{2}\right\rangle \equiv \sum_{\vec{r}} \vec{r}^{2}\left\langle O_{\vec{r}}^{2 \mathrm{D}}\right\rangle
$$

To evaluate this, we first rewrite Eq. (36) as

$$
\left\langle O_{\vec{r}}^{2 \mathrm{D}}\right\rangle=-\delta_{\vec{r}, \overrightarrow{0}}+\frac{1}{A} \int d^{2} \vec{k} p_{\vec{k}} e^{i \vec{k} \cdot \vec{r}},
$$

where the integral now runs over the entire Brillouin zone. We then note that $\vec{r}^{2} e^{i \vec{k} \cdot \vec{r}}=-\nabla_{\vec{k}}^{2} e^{i \vec{k} \cdot \vec{r}}$, integrate by parts in Eq. (38) so as to make $\nabla_{\vec{k}}^{2}$ act on $p_{\vec{k}}$, and use the identity $\sum_{\vec{r}} e^{i \vec{k} \cdot \vec{r}}=2 A \delta^{2}(\vec{k})$, to obtain $\left\langle\vec{r}^{2}\right\rangle=-2\left(\nabla_{\vec{k}}^{2} p_{\vec{k}}\right)_{\vec{k}=\overrightarrow{0}}=24 \pi \tau\left(J_{1}^{2}+J_{2}^{2}+J_{1} J_{2}\right) / J$. This shows that the spatial extent of $\left\langle O_{\vec{r}}^{2 \mathrm{D}}\right\rangle$ grows as $\sqrt{\tau}$ for large $\tau$. [Eq. (17) shows that we get the same behavior in 1D.] Finally, we can get an idea of the spatial anisotropy of $\left\langle O_{\vec{r}}^{2 \mathrm{D}}\right\rangle$ by computing

$$
\left\langle\vec{r}^{2}\right\rangle_{\theta} \equiv \sum_{\vec{r}}(x \cos \theta+y \sin \theta)^{2}\left\langle O_{\vec{r}}^{2 \mathrm{D}}\right\rangle,
$$

where $\vec{r}=(x, y)$, and $\theta$ denotes a direction along which the spatial extent is being calculated. By writing $(x \cos \theta+y \sin \theta)^{2} e^{i \vec{k} \cdot \vec{r}}=-\left(\cos \theta \partial / \partial k_{x}+\right.$ $\left.\sin \theta \partial / \partial k_{x}\right)^{2} e^{i \vec{k} \cdot \vec{r}}$, we can prove that $\left\langle\vec{r}^{2}\right\rangle_{\theta}=6 \pi \tau\left[\left(J_{1}-\right.\right.$ $\left.\left.J_{2}\right) \cos \theta+\sqrt{3}\left(J_{1}+J_{2}\right) \sin \theta\right]^{2} / J$. We see that $\left\langle\vec{r}^{2}\right\rangle_{\theta}$ has a marked dependence on $\theta$; in fact, it vanishes in the direction given by $\theta=\tan ^{-1}\left[\left(J_{2}-J_{1}\right) / \sqrt{3}\left(J_{2}+J_{1}\right)\right]$, and is maximum in the perpendicular direction. These statements should be interpreted with some care; $\left\langle\vec{r}^{2}\right\rangle_{\theta}$ may be small for some value of $\theta$ either due to a cancellation between positive and negative correlations or because $\left\langle O_{\vec{r}}^{2 \mathrm{D}}\right\rangle$ is small in that direction.

We note that the sum rule discussed in Sec. IIC is also valid in $2 \mathrm{D}$, and we get $\sum_{\vec{r}}\left\langle O_{\vec{r}}^{2 \mathrm{D}}\right\rangle=-1+2 p_{\overrightarrow{0}}=1$ regardless of how $J_{3}$ is varied from $-\infty$ to $\infty$.

\section{Entropy}

As discussed in Sec. ЏB, for each momentum $\vec{k}$, the final density matrix is effectively diagonal, with entries $1-p_{\vec{k}}$ and $p_{\vec{k}}$. The density matrix of the entire system takes the product form $\rho=\bigotimes \rho_{\vec{k}}$. The von Neumann entropy density corresponding to this state is given by

$$
s=-\frac{1}{A} \int d^{2} \vec{k}\left[\left(1-p_{\vec{k}}\right) \ln \left(1-p_{\vec{k}}\right)+p_{\vec{k}} \ln p_{\vec{k}}\right],
$$

where the integral again goes half the Brillouin zone. Let us now consider the dependence of this quantity on the quenching time scale $\tau \underline{12}$. If $\tau$ is very small, the system stays in its initial state and $p_{\vec{k}}$ will be close to 1 for all values of $\vec{k}$; for the same reason, $\left\langle O_{\overrightarrow{0}}\right\rangle$ will remain close to 1 . If $\tau$ is very large, the system makes a transition to the final ground state for all momentum except near the line described in Eq. (27). Hence $p_{\vec{k}}$ will be close to 0 for all $\vec{k}$ except near that line, and $\left\langle O_{\overrightarrow{0}}\right\rangle$ will be close to -1 . In both these cases, the entropy density will be small. We therefore expect that there will be an intermediate region of values of $\tau$ in which $s$ will show a maximum and $\left\langle O_{\overrightarrow{0}}\right\rangle$ will show a crossover from -1 to 1 . A plot of $s$ and as a function of $J \tau$ and $\alpha$, shown in Fig. 8 confirms this expectation. We find that the entropy reaches a maximum for the intermediate value of $J \tau$ where $\left\langle O_{\overrightarrow{0}}\right\rangle$ crosses over from -1 to 1 for all values of $\alpha$.

\section{EXTENDED KITAEV MODEL}

The extended Kitaev model, described by $H_{2}$ (Eq. (2)), can also be mapped, using the Majorana transformation given by Eq. (19), to a Fermionic Hamiltonian

$$
H_{1}^{\prime}=i J_{4} \sum_{(j, l) \in A}\left[a_{j, l} a_{j+2, l}-b_{j, l+1} b_{j+2, l+1}\right]+H_{2 \mathrm{D}},
$$

where $H_{2 \mathrm{D}}$ is given by Eq. (20). We note that in this model, just as for $H_{2 \mathrm{D}}, D_{n}$ commutes with all the terms in the Hamiltonian and the ground state corresponds to $D_{n}=1$ for all links of the honeycomb lattice. Thus, in 


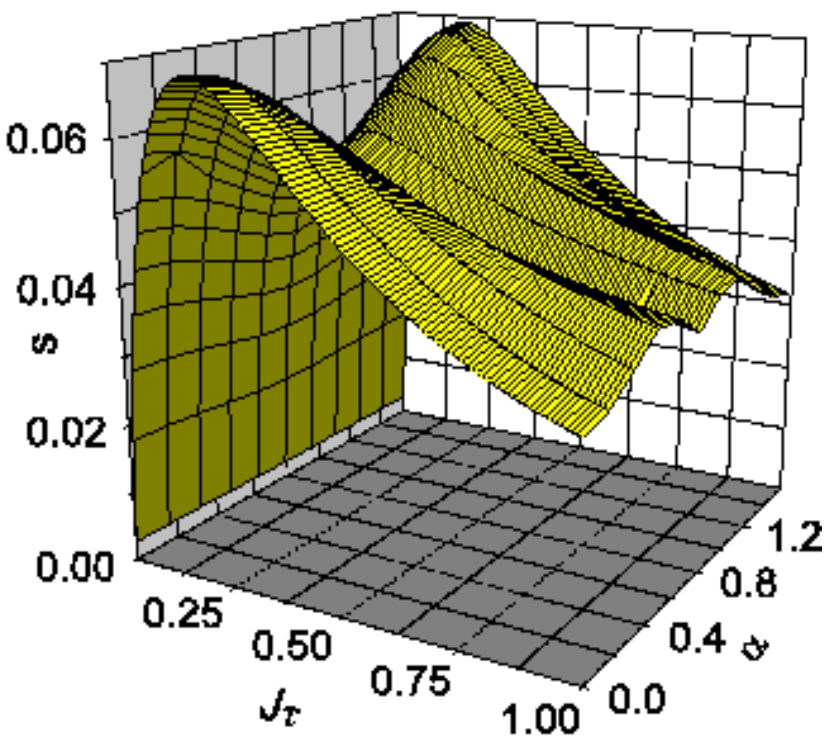

FIG. 8: Plot of the entropy density $s$ as a function of $J \tau$ and $\alpha=\tan ^{-1}\left(J_{2} / J_{1}\right)$. The entropy density peaks when $\left\langle O_{\overrightarrow{0}}\right\rangle$ crosses from -1 to 1 as discussed in the text.

momentum space, $H_{1}^{\prime}$ reduces to a bilinear 2 by 2 matrix Hamiltonian $H_{2}^{\prime}=\sum_{\vec{k}} \psi(\vec{k})^{\dagger} H_{3}^{\prime}(\vec{k}) \psi(\vec{k})$, where

$$
\begin{aligned}
H_{3}^{\prime}(\vec{k})= & 2\left\{\left[J_{1} \sin \left(\vec{k} \cdot \vec{M}_{1}\right)-J_{2} \sin \left(\vec{k} \cdot \vec{M}_{2}\right)\right] \sigma^{1}\right. \\
& +\left[J_{3}+J_{1} \cos \left(\vec{k} \cdot \vec{M}_{1}\right)+J_{2} \cos \left(\vec{k} \cdot \vec{M}_{2}\right)\right] \sigma^{2} \\
& \left.-J_{4} \sum_{k} \sin \left(\sqrt{3} k_{x}\right) \sigma^{3}\right\} .
\end{aligned}
$$

This can be diagonalized to obtain the energy eigenvalues

$$
\begin{aligned}
E_{\vec{k}}^{\prime \pm}= & \pm 2\left(J_{4}^{2} \sin ^{2}\left(\sqrt{3} k_{x}\right)+\left[J_{3}+J_{1} \cos \left(\vec{k} \cdot \vec{M}_{1}\right)\right.\right. \\
& \left.+J_{2} \cos \left(\vec{k} \cdot \vec{M}_{2}\right)\right]^{2}+\left[J_{1} \sin \left(\vec{k} \cdot \vec{M}_{1}\right)\right. \\
& \left.\left.-J_{2} \sin \left(\vec{k} \cdot \vec{M}_{2}\right)\right]^{2}\right)^{1 / 2}
\end{aligned}
$$

Note that the presence of a non-zero $J_{4}$ introduces a gap in the spectrum (except when $\sqrt{3} k_{x}=n \pi$ ) for all values of $J_{1}, J_{2}$ and $J_{3}$. Thus the quench of $J_{4}\left(J_{4}=J(t / \tau)\right)$ carries the system through a critical point at $t=0$ provided $\left|J_{1}-J_{2}\right| \leq J_{3} \leq\left(J_{1}+J_{2}\right)$.

The probability $p_{\vec{k}}$ of defect formation in such a quench, where the system evolves according to LandauZenner dynamics, can be read off from Eqs. (42,43) as

$$
p_{\vec{k}}=e^{-\pi \tau\left(E_{\vec{k}}^{\prime} \pm\right)^{2}\left|J_{4}=0 /\right| 2 J \sin \left(\sqrt{3} k_{x}\right) \mid} .
$$

The density of defects is thus given by $n=\int d^{2} \vec{k} p_{\vec{k}} / A$, where the integral is taken over half the Brillouin zone

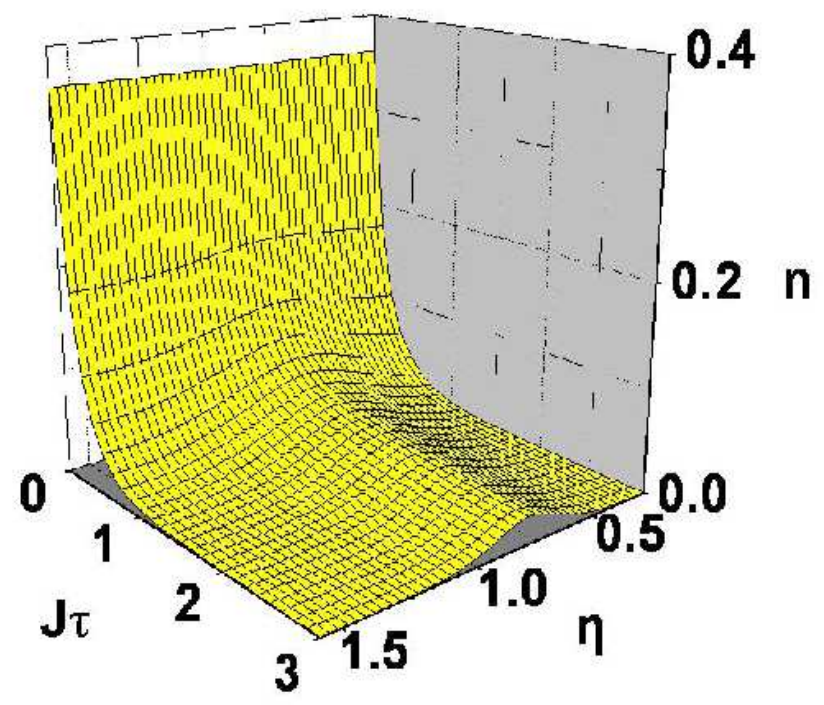

FIG. 9: Plot of the defect density as a function of $\eta=J_{3} / J_{1}$ and $J \tau$ for $J_{1}=J_{2}=J$.

defined by the triangle with vertices lying at $\left(k_{x}, k_{y}\right)=$ $(2 \pi / \sqrt{3}, 0),(0,2 \pi / 3),(0,-2 \pi / 3)$ and $A$ is the area of this region. A plot of the defect density as a function of the quench rate $\tau$ and $\eta=J_{3} / J_{1}$ for $J_{1}=J_{2}=J$ is shown in Fig. 9. Note that for large quench time $\tau$, the maximum contribution to the quench comes from around the momentum $\vec{k}_{0}=\left(k_{x 0}, k_{y 0}\right)$ for which $\left.E_{\vec{k}_{0}}^{\prime \pm}\right|_{J_{4}=0}$ vanishes. Around this point $p_{\vec{k}} \sim \exp \left[-\pi J \tau \sum_{\alpha, \beta=x, y} f_{\alpha \beta}\left(\vec{k}_{0}\right)(\vec{k}-\right.$ $\left.\left.\vec{k}_{0}\right)_{\alpha}\left(\vec{k}-\vec{k}_{0}\right)_{\beta}\right]$ so that $n \sim 1 / \tau$ in accordance with the prediction of the general formula Eq. (32) for $d=m=2$ and $\nu=z=1$.

Next, we look at the defect correlation functions for the extended Kitaev model. To this end, we define the operator

$$
O_{\vec{r}}^{\text {ext }}=i\left(a_{\vec{n}} a_{\vec{n}+\vec{r}}-b_{\vec{n}} b_{\vec{n}+\vec{r}}\right)
$$

and consider its expectation value for $\vec{r} \neq \overrightarrow{0}$. Here $\vec{r}=\left(\sqrt{3} n_{1}+\sqrt{3} n_{2} / 2,3 n_{2} / 2\right)$ (with integers $n_{1}$ and $\left.n_{2}\right)$ specifies the sites of the honeycomb lattice. (For $\vec{r}=\overrightarrow{0}$, $O_{\vec{r}}^{\text {ext }}$ vanishes since $a_{\vec{n}}^{2}=b_{\vec{n}}^{2}=1$ ).

For $J_{4} \rightarrow \mp \infty$, the model reduces to a set of decoupled chains involving Majorana fermions on nearest neighbor sites. For this model, it is known ${ }^{30}$ that for $\vec{r} \neq \overrightarrow{0}$,

$$
\left\langle O_{\vec{r}}^{\text {ext }}\right\rangle=\mp \delta_{n_{2}, 0} \frac{2}{\pi n_{1}}\left[(-1)^{n_{1}}-1\right]
$$

in the ground states for $J_{4} \rightarrow \mp \infty$ respectively. For generic values of $J_{4}$ and for a mixed final state after the 
quench characterized by a defect probability $p_{\vec{k}}$, we find

$$
\begin{aligned}
\left\langle O_{\vec{r}}^{\text {ext }}\right\rangle= & -\frac{8}{N} \sum_{\vec{k}}\left\langle a_{\vec{k}}^{\dagger} a_{\vec{k}}-b_{\vec{k}}^{\dagger} b_{\vec{k}}\right\rangle \sin (\vec{r} \cdot \vec{k}) \\
= & \delta_{n_{2}, 0} \frac{2}{\pi n_{1}}\left[(-1)^{n_{1}}-1\right] \\
& +\frac{4}{A} \int d^{2} \vec{k} \operatorname{sgn}\left[\sin \left(\sqrt{3} k_{x}\right)\right] p_{\vec{k}} \sin (\vec{r} \cdot \vec{k}) .
\end{aligned}
$$

The sign of $\sin \left(\sqrt{3} k_{x}\right)$ appears in Eq. (47) because for $J_{4} \rightarrow \infty$, the ground state of Eq. (42) has $\left\langle a_{\vec{k}}^{\dagger} a_{\vec{k}}-b_{\vec{k}}^{\dagger} b_{\vec{k}}\right\rangle= \pm 1$ depending on whether $\sin \left(\sqrt{3} k_{x}\right)>$ 0 or $<0$ respectively.

To obtain an analytical understanding of the nature of the correlation function, we look at the case where $J_{1}=J_{2}=J, J_{3}=\eta J$ and $J \tau \rightarrow \infty$. Note that one needs the condition $0 \leq \eta \leq 2$ for the system to pass through a gapless (critical) point during the quench. In this case, the main contribution to the last term of the correlation function $\left\langle O_{\vec{r}}^{\text {ext }}\right\rangle$ in Eq. (47) comes from $\vec{k}=$ $\vec{k}_{0}=\left((2 / \sqrt{3}) \cos ^{-1}(-\eta / 2), 0\right)$ where $p_{\vec{k}=\vec{k}_{0}}=1$. Thus for $J \tau \rightarrow \infty$ one gets

$$
\left\langle O_{\vec{r}}^{\text {ext }}\right\rangle \simeq \sin \left[\left\{2 n_{1}+n_{2}\right\} \cos ^{-1}\left(\frac{-\eta}{2}\right)\right]
$$

where we have omitted the first term (proportional to $\left.\delta_{n_{2}, 0}\right)$ in Eq. (47). Eq. (48) clearly brings out the dependence of the spatial anisotropy of the defect correlation function as a function of $\eta$. In particular, for $\eta=0$, $\left\langle O_{\vec{r}}^{\text {ext }}\right\rangle \sim \sin \left\{\left(n_{1}+n_{2} / 2\right) \pi\right\}$, so that its sign alternates between sites with odd and even values of $n_{1}$ (if $n_{2}$ is odd). Similarly, for $\eta=2,\left\langle O_{\vec{r}}^{\text {ext }}\right\rangle \sim \sin \left\{\left(2 n_{1}+n_{2}\right) \pi\right\} \sim 0$. Such a behavior of the correlation function is qualitatively supported by the numerical computation of $\left\langle O_{\vec{r}}^{\text {ext }}\right\rangle$ for $J_{1}=J_{2}=J, J_{3}=\eta J$ and $J \tau=3$ as shown in Fig. 10. We find that for $\eta=0$ (top left plot of Fig. 10), it alternates between odd and even $n_{1}$ sites, while for $\eta$ close to 2 (bottom right plot in Fig. 10), the correlation function is much smaller than for $\eta=0$.

Finally, we compute the entropy generated due to such a quench process given by Eq. (40) where $p_{\vec{k}}$ is given by Eq. (44). A plot of the entropy density as a function of $J \tau$ and $\alpha=\tan ^{-1}\left(J_{2} / J_{1}\right)$ with $J_{1}=J_{3}=J$ is shown in Fig. 11. Once again we find, similar to that in the Kitaev model, that the entropy density peaks for intermediate value of $\tau$.

\section{DISCUSSION}

In conclusion, we have studied the quench dynamics of the Kitaev model in 1D and 2D and the extended Kitaev model in 2D. For the 1D Kitaev model and the 2D extended Kitaev model, we have shown that the defect
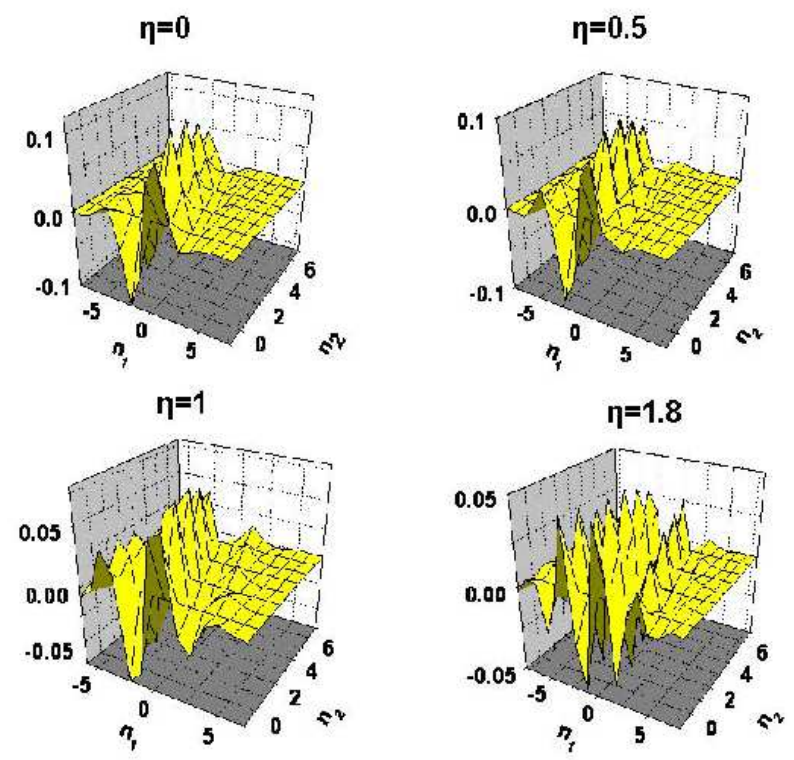

FIG. 10: Plot of the defect correlation function (sans the first term with the delta function peak in Eq. (47)) with $J \tau=3$ and $J_{1}=J_{2}=J$ for several representative values of $\eta=$ $J_{3} / J_{1}$. See text for details.

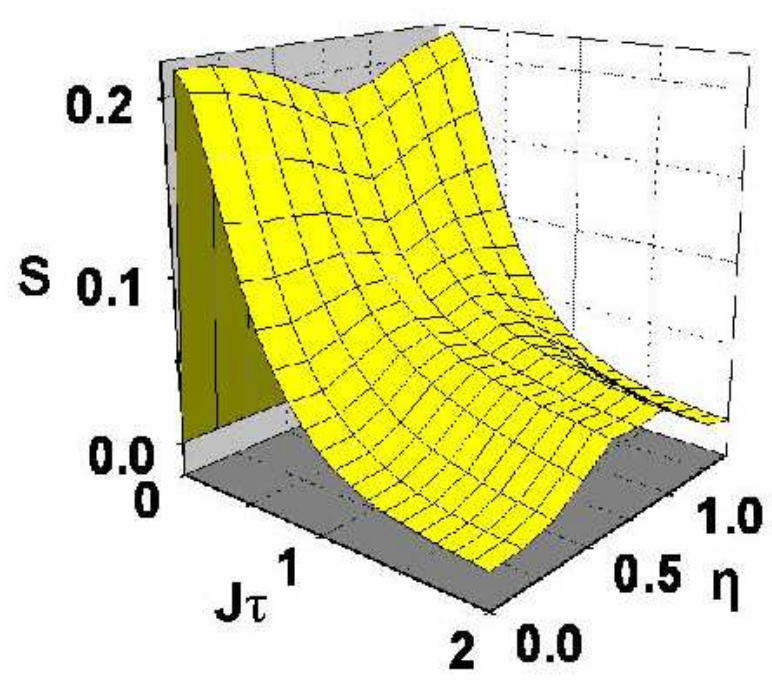

FIG. 11: Plot of the entropy density $s$ as a function of quench time $\tau$ and $\eta=J_{3} / J_{1}$.

density scales as $1 / \tau^{d / 2}$ with the quench time $\tau$, in accordance with the general results of Ref. 4 . For the $2 \mathrm{D} \mathrm{Ki-}$ atev model, where the quench takes the system through a gapless line, we found that the scaling of the defect density with $\tau$ changes due to the presence of a critical line instead of a critical point. In this context, we have presented a general formula for the quench rate dependence of the defect density for a $d$ dimensional system when the quench takes such a system through a $d-m$ dimensional critical surface. We have also computed the 
defect correlation function for such quenches by an exact computation of all independent non-zero spin correlation functions in the defect ground state. In $d=2$, we have found that the defect correlation function exhibit spatial anisotropy and studied the dependence of this anisotropy with the system parameter. Finally, we have computed the entropy generated in such processes and have shown that the entropy peaks approximately at values of the quench rate for which the defect correlation function changes from -1 to 1 .

There have been proposals for experimentally realizing the Kitaev model in systems of ultracold atoms and molecules trapped in optical lattices ${ }^{31}$. If this can be done, the evolution of the defect correlations with various parameters (such as $J_{2} / J_{1}$ as shown in Fig. 7) can, in principle, be experimentally detected by spatial noise correlation measurements as pointed out in Ref. 32 .
Finally, we would like to note that the quench dynamics of the $X X Z$ spin-1/2 chain has been recently studied with the Hamiltonian being varied along a line in parameter space where the model is critical 33 . In momentum space, the model only has a finite number of critical points, but the system stays close to those critical points for a long time. This is a different situation from the one that we have analyzed in Sec. III where there is a line of critical points in momentum space; hence our results for the scaling of the defect density are not applicable to the work in Ref. 33 .

We thank Amit Dutta and Anatoly Polkovnikov for stimulating discussions and several important suggestions. DS thanks DST, India for financial support under the project SR/S2/CMP-27/2006.
1 S. Sachdev, Quantum Phase Transitions (Cambridge University Press, Cambridge, 1999).

2 T. W. B. Kibble, J. Phys. A 9, 1387 (1976); W. H. Zurek, Nature (London) 317, 505 (1985).

3 B. Damski, Phys. Rev. Lett. 95, 035701 (2005).

4 A. Polkovnikov, Phys. Rev. B 72, 161201(R) (2005).

5 A. Polkovnikov and V. Gritsev, arXiv:0706.0212 2 , to appear in Nature Physics.

6 The rate of defect production might change if the quench takes the system through some special multicritical point. See Ref. 12 for a discussion of this issue.

7 K. Sengupta, S. Powell, and S. Sachdev Phys. Rev. A 69, 053616 (2004).

8 J. Dziarmaga, Phys. Rev. Lett. 95, 245701 (2005), and Phys. Rev. B 74, 064416 (2006).

${ }^{9}$ P. Calabrese and J. Cardy, J. Stat. Mech: Theory Expt P04010 (2005), and Phys. Rev. Lett. 96, 136801 (2006).

10 A. Das, K. Sengupta, D. Sen, and B. K. Chakrabarti, Phys. Rev. B 74, 144423 (2005).

11 R. W. Cherng and L. Levitov, Phys. Rev. A 73, 043614 (2006).

12 V. Mukherjee, U. Divakaran, A. Dutta, and D. Sen, Phys. Rev. B 76, 174303 (2007).

13 B. Damski and W. H. Zurek, Phys. Rev. A 73063405 (2006).

14 T. Caneva, R. Fazio, and G. E. Santoro, Phys. Rev. B 76, 144427 (2007).

15 F. M. Cucchietti, B. Damski, J. Dziarmaga, and W. H. Zurek, Phys. Rev. A 75, 023603 (2007).

16 For a review see I. Bloch, J. Dalibard, and W. Zwerger, arXiv:0704.3011v2, to appear in Rev. Mod. Phys.

17 L. E. Sadler, J. M. Higbie, S. R. Leslie, M. Vengalattore, and D. M. Stamper-Kurn, Nature (London) 443, 312 (2006).
18 A. Kitaev, Ann. Phys. 321, 2 (2006).

19 X.-Y. Feng, G.-M. Zhang, and T. Xiang, Phys. Rev. Lett. 98, 087204 (2004).

${ }^{20}$ H.-D. Chen and Z. Nussinov, arXiv:cond-mat/0703633v5 (unpublished).

21 Z. Nussinov and G. Ortiz, arXiv:0709:2717v3 (unpublished)

22 G. Baskaran, S. Mondal, and R. Shankar, Phys. Rev. Lett. 98, 247201 (2007).

23 K. P. Schmidt, S. Dusuel, and J. Vidal, Phys. Rev. Lett. 100, 057208 (2008).

24 A. Kitaev, Ann. Phys. 303, 2 (2003).

25 D.-H. Lee, G.-M. Zhang, and T. Xiang, Phys. Rev. Lett. 99, 196805 (2007).

${ }^{26}$ K. Sengupta, D. Sen, and S. Mondal, Phys. Rev. Lett. 100, 077204 (2008).

27 See for example, J. B. Kogut, Rev. Mod. Phys. 51, 659 (1979).

28 See for example, L. Landau and E. M. Lifshitz, Quantum Mechanics: Non-relativistic Theory, 2nd Ed. (Pergamon Press, Oxford, 1965).

29 See for example S. Suzuki and M. Okada in Quantum Annealing and Related Optimization Methods, Eds. by A. Das and B. K. Chakrabarti (Springer-Verlag, Berlin, 2005).

30 B. S. Shastry and D. Sen, Phys. Rev. B 55, 2988 (1997).

31 L.-M. Duan, E. Demler, and M. D. Lukin, Phys. Rev. Lett. 91, 090402 (2003); A. Micheli, G. K. Brennen, and P. Zoller, Nature Physics 2, 341 (2006).

${ }^{32}$ E. Altman, E. Demler, and M. D. Lukin, Phys. Rev. A 70, 013603 (2004).

${ }^{33}$ F. Pellegrini, S. Montangero, G. E. Santoro, and R. Fazio, arXiv:0801.4475 1 (unpublished). 